\title{
The Electric Field and Temperature Dependence of Conductance of Two-Dimensional Electron Gas in $\mathrm{AlGaN} / \mathrm{AlN} / \mathrm{GaN}$
}

\author{
L. Ardaravičius, O. Kiprijanovič and J. Liberis \\ Semiconductor Physics Institute, A. Goštauto 11, LT-01108, Vilnius, Lithuania \\ The two-dimensional gas in AlGaN/AlN/GaN heterostucture with a \\ very thin $(0.6 \mathrm{~nm})$ AlN spacer was investigated by conductivity relaxation \\ measurements in 86-300 K temperature range. The results show the presence \\ of two exponential relaxation processes characterized by different character- \\ istic time constants. Parameters of the fast and slow components of the \\ processes differently depend on the electric field and temperature. The fast \\ process is attributed to influence of the electric field on the barrier formed by \\ the spacer, while the slow process is attributed to the hot-electron capture \\ out of the channel followed by electron thermal release.
}

PACS numbers: 72.20.Jv, 73.40.Kp, 73.50.Fq

\section{Introduction}

One of the main objectives in explaining the hot electron transport in AlGaN/AlN/GaN two-dimensional electron gas (2DEG) channels is understanding of the drift velocity saturation magnitude $\left(\approx 1 \times 10^{7} \mathrm{~cm} / \mathrm{s}\right)$ at electric fields higher than $15 \div 20 \mathrm{kV} / \mathrm{cm}$, far below the theory predictions: $\approx 2 \div 2.5 \times 10^{7} \mathrm{~cm} / \mathrm{s}$ in the $40 \div 80 \mathrm{kV} / \mathrm{cm}$ range [1]. Some processes, that could influence 2DEG sheet density, have been pointed out which can be observed in nanosecond time scale of conductance relaxation. Among these are: (1) self heating and its cooling down after the electric field pulse [2]; (2) transfer and trapping of hot carriers from 2DEG channel to either AlN spacer or GaN buffer layer which contains high concentration of deep traps and subsequent release [3]; (3) electric field and temperature influence on spontaneous and piezoelectric polarization [4]. Trapping effects with characteristic times lying within $5 \div 10 \mu$ s have been indicated [5].

In this work, for better understanding of hot electron transport in 2DEG channels at high electric fields, conductivity relaxation in AlGaN/AlN/GaN channel after strong $1 \mathrm{~ns}$ wide electric pulse action was investigated in the temperature range from $80 \mathrm{~K}$ to $300 \mathrm{~K}$. 


\section{Experimental}

The samples were grown by metal-organic chemical vapor deposition (MOCVD) method at the University of California (Santa Barbara). The 2DEG channel was located in GaN in AlGaN/AlN/GaN heterostructure with an ultrathin $(0.6 \mathrm{~nm})$ AlN spacer. The $100 \mu \mathrm{m}$ width $2 \mathrm{DEG}$ channel had the electron density $9.78 \times 10^{12} \mathrm{~cm}^{-2}$ and mobility $1664 \mathrm{~cm}^{2} / \mathrm{Vs}$. Coplanar Ti/Al/Ti/Au electrodes were formed at $1080 \mathrm{~K}$; two $100 \times 100 \mathrm{~m}^{2}$ contact pads were separated with a gap $10 \mu \mathrm{m}$.

The 2DEG channel ohmic conductivity $\sigma_{0}$ increase with lowering the temperature is due to mobility $\mu$ increase [4]. The $I-V$ characteristics at high electric fields and conductivity relaxation measurements were carried out with circuit shown in Fig. 1. The high-field part consists of a generator with a charged line

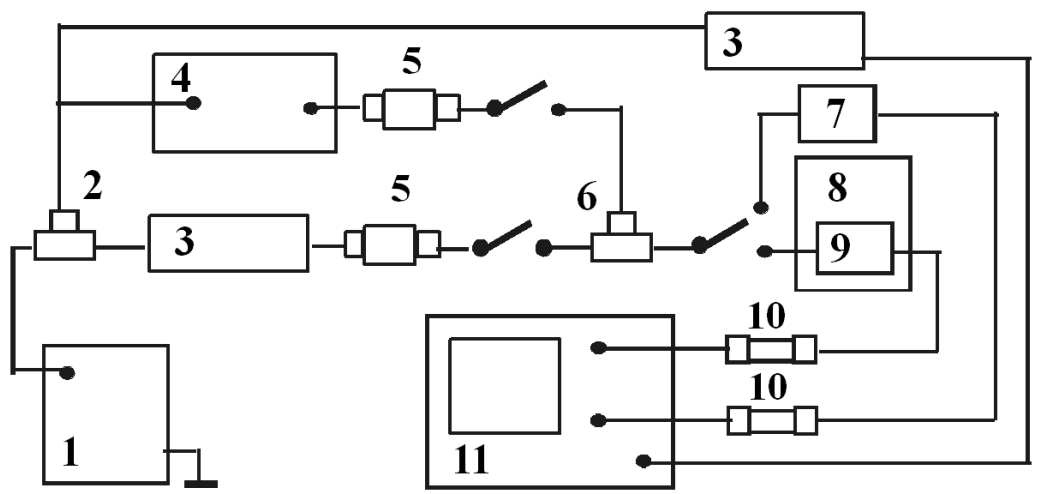

Fig. 1. Schematic circuit for measurements in micro- and nanosecond time scale.

and a power source (1). The high-field part consists of a generator with a charged line and a power source, synchronization tee (2) and delay line (3). A variable power attenuator (5) is used for high pulse amplitude control. The gauge resistor (7) value is chosen close to the sample resistance and is used to measure the incident high-voltage pulse amplitude fed to input of oscilloscope (11). Thin film attenuators (10) served as oscilloscope input protectors. Full information is obtained from the measurement of pulses transmitted through a sample (9) placed in cryostat (8). The low-field part contains the test generator (4) that provides $500 \mathrm{~ns}$ low-voltage pulses via bias-tee (6) for relaxation measurements.

\section{Experimental results}

Conductivity relaxation measurements with $2 \mathrm{~ns}$ (at base) high voltage electric pulse are presented in Fig. 2 as points. The conductivity relaxation was observed after the threshold of about $25 \div 30 \mathrm{kV} / \mathrm{cm}$. The results were approximated with exponentials by the following formula: 

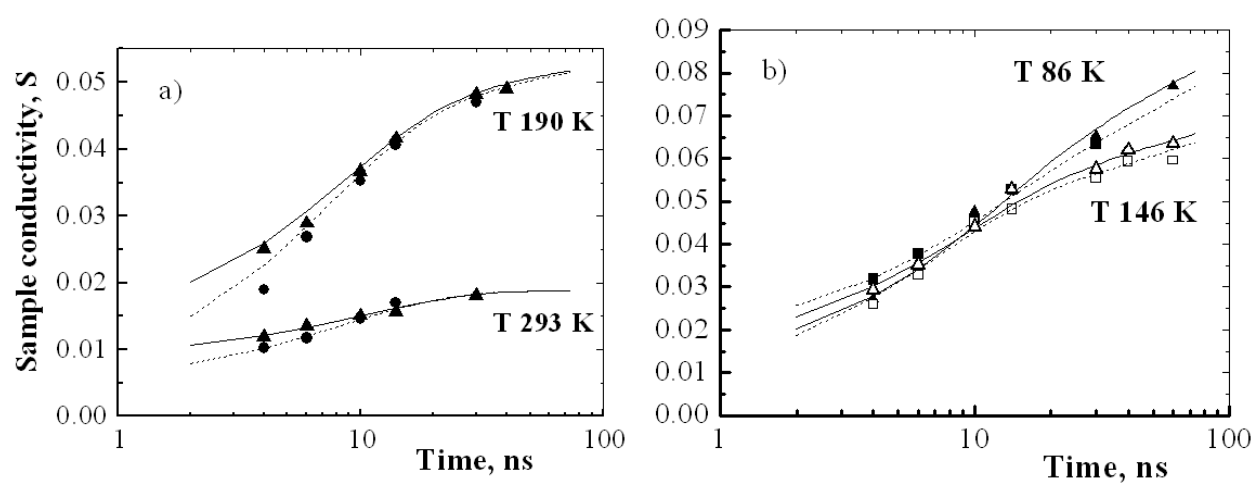

Fig. 2. Low field conductivity relaxation after high pulse action. (a) $T=190 \mathrm{~K}, 293 \mathrm{~K}$, (b) $T=86 \mathrm{~K}, 146 \mathrm{~K}$. Symbols — experiment, lines — exponential approximation (1) (solid $E=49 \mathrm{kV} / \mathrm{cm}$, dashed $E=59 \mathrm{kV} / \mathrm{cm}$ ).

$$
\sigma(t)=\left(\sigma_{0}-\sigma(0)\right)\left\{A_{1}\left[1-\exp \left(-t / \tau_{1}\right)\right]+A_{2}\left[1-\exp \left(-t / \tau_{2}\right)\right]\right\}+\sigma(0),
$$

where $\sigma(0)$ is the conductivity extrapolated to $t=0$ (Fig. 2). Our setup cannot measure the processes that are faster than about few nanoseconds. The fitting results are shown in Table. The conductivity relaxation process at high electric fields consists of two parts - slow part characterized by the amplitude $A_{2}$ and the time constant $\tau_{2}$ and fast part $\left(\tau_{1}, A_{1}\right)$.

TABLE

Fitting parameters in formula (1)

\begin{tabular}{c|c|c|c|c|c|c}
\hline \hline $\mathrm{T}[\mathrm{K}]$ & $E[\mathrm{kV} / \mathrm{cm}]$ & $A_{1}$ & $\tau_{1}[\mathrm{~ns}]$ & $A_{2}$ & $\tau_{2}[\mathrm{~ns}]$ & $\sigma(0)[\mathrm{S}]$ \\
\hline 293 & 49 & 0.9 & 10 & 0.1 & 18 & 0.009 \\
& 59 & 0.9 & 8 & 0.1 & 18 & 0.005 \\
190 & 49 & 0.8 & 8 & 0.2 & 40 & 0.0125 \\
& 59 & 0.8 & 7 & 0.2 & 40 & 0.005 \\
146 & 49 & 0.65 & 8 & 0.35 & 55 & 0.014 \\
& 59 & 0.65 & 6.5 & 0.35 & 65 & 0.007 \\
86 & 49 & 0.55 & 10 & 0.45 & 60 & 0.011 \\
& 59 & 0.45 & 9.5 & 0.55 & 70 & 0.018
\end{tabular}

\section{Discussion of the results}

The results of our measurements indicate the absence of conductivity relaxation process in ns time scale at low electric field at room temperature. The small enough sample conductivity $\sigma(0)$ obtained from extrapolation indicates the presence of relatively long (ns) rebuilding of the channel sheet concentration. Indeed, the ultrathin $0.6 \mathrm{~nm}$ thick AlN layer does not prevent the hot electrons from 
tunneling from GaN into the AlGaN layer at high electric fields. There is also possible influence on the layer strain changing piezoelectric polarization, which modifies barrier properties. We attribute the fast process corresponding to the time constant $\tau_{1}$ to the influence of electric field on the shape of the barrier (the barrier recovers its properties after the pulse). Also, it is important to note that trap levels in the channel are occupied at equilibrium. The empty traps are located outside the channel in the barrier layers. Therefore, real space transfer of hot electrons is a prerequisite for the electrons to be captured by empty traps. We assign the process with a long time constant $\tau_{2}$ to hot electron capture out of the channel followed by electron thermal release.

By analyzing the parameters in Table, we see that with the decrease in ambient temperature more electrons out from the channel are captured by the empty traps. This is indicated by increase in constant $\tau_{2}$ value and amplitude $A_{2}$. The rest $3 \mathrm{D}$ electrons move in parallel with the channel electrons. The mobility of the former is lower than the channel mobility and increases with lowering temperature. The latter fact can be used for explanation of the conductance increase with the electric field at $86 \mathrm{~K}$ temperature, as compared to other temperatures. With decreasing ambient temperature, from $295 \mathrm{~K}$ to $86 \mathrm{~K}$, due to capture by empty traps, less and less electrons are drifting while the amount of electrons leaving the channel weakly depends on temperature. Relaxation methods could be employed for analyzing AlN and other barrier properties in AlGaN/AlN/GaN heterostructures applying high electric fields of nanosecond duration, therefore they can provide more features of 2DEG drift velocity estimation at high electric fields.

\section{References}

[1] M. Ramonas, A. Matulionis, J. Liberis, L. Eastman, X. Chen, Y.J. Sun, Phys .Rev. B 71, 075324 (2005).

[2] S. Vitusevich, S. Daniliuk, N. Klein, M. Petrychuk, A. Avksentyev, V. Sokolov, V. Kochelap, A. Beliaev, V. Tilak, J. Smart,. A. Vertiatchich, L. Eastman, Appl. Phys. Lett. 82, 748 (2003).

[3] A.P. Zhang, L.B. Rowland, E.B. Kaminsky, V. Tilak, J.C. Grande, J. Teetsov, A. Vertiatchikh, L.F. Eastman, J. Electron. Mater. 32, 388 (2003).

[4] B. Shen, T. Someaya, Y. Arakawa, Appl. Phys. Lett. 76, 2746 (2000).

[5] O. Mitrofanov, M. Manfra, Appl. Phys. Lett. 84, 422 (2004). 